\title{
Percepção de risco da utilização e descarte de embalagens de agroquímicos em hortas, em Santarém, Pará
}

\author{
Éder Bruno Rebelo da Silva ${ }^{1^{*}}$, Gilbson Santos Soares ${ }^{2}$, Welligton Conceição da Silva ${ }^{3}$, Maria Roseane Pereira \\ dos Santos ${ }^{4}$
}

DOI: https://doi.org/10.35699/2447-6218.2020.25062

\begin{abstract}
Resumo
O objetivo neste estudo foi analisar a percepção de risco no uso de agroquímicos quanto a utilização e descarte das embalagens vazias em hortas no município de Santarém, Pará. O estudo foi realizado no município de Santarém, Pará, com o intuito de verificar a percepção dos produtores que utilizam agroquímicos em hortas, assim, foram realizadas entrevistas in loco com aplicação de questionário contendo 10 perguntas de caráter aberto e fechado, sendo avaliadas 7 comunidades produtoras de hortaliças (Área Verde, Cristo Rei, Floresta, Jutaí, Mararu, Tabocal e Urumanduba), totalizando 30 agricultores. Analisando os resultados, observou-se que todos os produtores respeitam o período de carência e grande parte faz uso de métodos convencionais, seguindo principalmente as instruções do rótulo, observou-se também que a maioria dos produtores recebe orientação de profissionais com conhecimento técnico sobre o assunto. Percebeu-se que o agroquímico mais adotado foi o inseticida, além disso, apenas uma pequena parte dos produtores utiliza equipamento de proteção individual completo e a maioria realiza a lavagem das roupas após a utilização, entretanto, não realizam a tríplice lavagem das embalagens vazias. Por fim, pode-se perceber que grande parte dos produtores não tem conhecimento sobre o posto de coleta e descartam a embalagem dos agroquímicos principalmente no lixo. Diante do exposto, conclui-se que os produtores de hortaliças do município de Santarém não fazem o descarte correto das embalagens vazias de agroquímicos, descartando-as em ambiente inadequado, sendo que nenhum produtor devolveu a embalagem no posto de coleto conforme preconiza a legislação brasileira.
\end{abstract}

Palavras-chave: Ambiente. Defensivos agrícolas. Inseticida.

\section{Risk perception of the use and disposal of agrochemical packaging in vegetable gardens, in Santarém, Pará}

\begin{abstract}
The objective of this study was to analyze the perception of risk in the use of agrochemicals regarding the use and disposal of empty packaging in vegetable gardens in the municipality of Santarém, Pará. The study was carried out in the municipality of Santarém, Pará, in order to verify the perception of producers who use agrochemicals in vegetable gardens, thus, were evaluated on the spot with the application of a questionnaire containing 10 open and closed questions, with 7 vegetable producing communities being evaluated (Área Verde, Cristo Rei, Floresta, Jutaí, Mararu, Tabocal and Urumanduba ), totaling 30 farmers. Analyzing the results, it was observed that all producers respect the grace period and a large part makes use of conventional methods, following mainly as label instructions, it was also observed that most producers receive guidance from professionals with technical knowledge on the subject. It
\end{abstract}

${ }^{1}$ Centro Universitário Luterano de Santarém, Santarém, PA. Brasil.

http://orcid.org/ 0000-0002-2964-8471

${ }^{2}$ Instituto Federal de Educação, Ciência e Tecnologia e Centro Universitário Luterano de Santarém, Santarém, PA. Brasil.

http://orcid.org/0000-0001-8810-1954

${ }^{3}$ Universidade Federal Rural da Amazônia, Belém, PA. Brasil.

http://orcid.org/ 0000-0001-9287-0465

${ }^{4}$ Universidade Federal do Oeste do Pará, Santarém, PA. Brasil.

http://orcid.org/ 0000-0002-3721-6564

*Autor para correspondência: eder.b.rebelo@gmail.com

Recebido para publicação em 31 de agosto de 2020. Aceito para publicação em 18 de novembro de 2020 .

e-ISSN: 2447-6218 / ISSN: 2447-6218. Atribuição CC BY. 
was noticed that the most widely used agrochemical was the insecticide, besides, only a small part of the producers used complete personal protective equipment and most of them wash the after use, however, they do not perform a triple washing of the empty packaging. Finally, it can be seen that most producers are unaware of the collection point and discard the packaging of agrochemicals mainly in the trash. In view of the above, it is concluded that vegetable producers in the municipality of Santarém do not correctly dispose of empty agrochemical packages, discarding them in an advanced environment, and no producer returned a package at the collection point as recommended by Brazilian legislation.

Keywords: Environment. Insecticide. Pesticides.

\section{Introdução}

O cultivo de hortaliças através das conhecidas hortas é uma prática que ocorre a bastante tempo, sendo para muitas famílias a principal fonte de renda (Ferreira e Cepolini, 2014). Para Pantoja et al. (2013) a horta é um laboratório vivo, dessa forma toda interação química e biológica vai causar uma reação, seja ela benéfica ou danosa.

Nesse contexto, os agroquímicos são substâncias químicas artificiais utilizadas na agricultura e pecuária, sendo tóxicos em graus variados ao homem, animais e meio ambiente. Sua principal função é a proteção vegetal contra-ataques de pragas e moléstias, tais como bactérias, fungos, insetos e no controle das chamadas ervas daninhas ou plantas invasoras (Lopes e Albuquerque, 2018).

O Brasil por ser um grande produtor agrícola apresenta altas taxas de intoxicação por agroquímico, seja no meio rural ou urbano, devido principalmente à falta de conhecimentos de manejo (Lara et al., 2019). Os agroquímicos podem trazer riscos à saúde, e dependendo do tempo de exposição ao químico, além da quantidade utilizada, isso vai se agravar ainda mais, ou seja, a falta de conhecimento ocasiona a superdosagem e é agravada pelo uso parcial ou nulo dos Equipamentos de Proteção Individual (EPI) (Santos et al., 2017).

O uso do EPI torna seguro a aplicação de agroquímicos, entretanto há grandes evidências que um elevado número de agricultores não faz o uso desses equipamentos de proteção (Sousa et al., 2016). Segundo Alves (2013) os EPIs existem para proteger a saúde do trabalhador e devem ser testados e aprovados pelas autoridades competentes objetivando comprovar sua eficácia, desse modo, devem ser utilizados para garantir a integridade do aplicador e proteger contra contaminações ao meio externo.

Na aplicação de agroquímicos em hortas a preocupação extra é quando ao descarte das embalagens vazias, o principal motivo da destinação correta das embalagens é diminuir o risco a saúde e a contaminação do meio ambiente (Bernardi et al., 2018).

A grande demanda na produção de hortaliças faz com que o produtor busque otimizar sua produção utilizando agroquímicos, o que favorece o aumento de recipientes para descarte, estima-se que o Brasil produz para acompanhar essa demanda 115 milhões de embalagens para armazenar 250 toneladas de agroquímicos (Oliveira e Sabonaro, 2016).

A utilização e o manejo inadequado de defensivos agrícolas podem ocasionar impactos ambientais e prejuízos à saúde dos seres humanos e dos animais (Lopes e Albuquerque, 2018), neste contexto, justifica-se este estudo pela necessidade de identificar os principais problemas relacionados ao modo de aplicação, utilização de EPIs, local de descarte das embalagens vazias e conhecimento dos produtores sobre o posto de coleta. Neste estudo, adotamos a hipótese de que os produtores de hortaliças do município de Santarém fazem o descarte correto das embalagens vazias de agroquímicos.

Com base nessas informações, o objetivo neste trabalho foi analisar a percepção de risco no uso de agroquímicos quanto à utilização e descarte das embalagens vazias em hortas no município de Santarém, Pará.

\section{Material e métodos}

\section{Local}

Este é um estudo de caráter descritivo, a pesquisa foi realizada com agricultores de hortas residentes no município de Santarém, Pará $\left(02^{\circ} 26^{\prime} 35^{\prime \prime} \mathrm{S}\right.$ e $54^{\circ} 42^{\prime}$ 30 " W), situado no oeste paraense, mesorregião médio Amazonas, no período de dezembro de 2019 a janeiro de 2020.

\section{Seleção da amostra}

A fração de um todo é definida como amostra, ou seja, uma pequena parcela de uma população (Bolfarine e Bussad, 2004). Neste estudo, a amostra é composta, exclusivamente por produtores de hortaliças do município de Santarém, Pará.

Foram avaliadas 7 comunidades produtoras de hortaliças (Área Verde, Cristo Rei, Floresta, Jutaí, Mararu, Tabocal e Urumanduba), sendo entrevistados 30 agricultores, desse modo, foram aplicados 30 questionários. A abordagem foi cautelosa, devido ao receio de ser um órgão de fiscalização, por parte dos produtores. 
Percepção de risco da utilização e descarte de embalagens de agroquímicos em hortas, em Santarém, Pará

\section{Roteiro e coleta de dados}

Para mensurar a percepção do produtor a respeito do período de carência, observando se estes recebem alguma instrução quanto ao manejo de agroquímicos e qual era responsável pela transmissão dessas informações, bem como verificar qual o agroquímico foi utilizado na horta, como se deu a utilização dos Equipamentos de Proteção Individual (EPI), se realizam a tríplice lavagem, onde estes costumavam descartar as embalagens vazias e se possuíam conhecimento sobre o posto de coleta.

Com o intuito de verificar a percepção do produtor, o método de coleta de dados foi a entrevista com questionário semiestruturado contendo 10 perguntas de caráter aberto e fechado, o questionário aberto possibilitava ao produtor a resposta livre, já o fechado dava direito a resposta de "Sim" ou "Não" e de múltipla escolha, desenvolvido em feiras e em visitas in loco nas propriedades.

As perguntas realizadas no questionário foram se o produtor respeita o período de carência; o tipo de cultivo; se segue as instruções de aplicação do rótulo, vendedor, engenheiro agrônomo/técnico ou outro; se já recebeu instruções sobre o uso de agroquímicos; se sim, de quem; qual o agrotóxico é mais utilizado na propriedade; se faz uso de EPI (equipamento de proteção individual); se a roupa é lavada após a utilização; se possuem conhecimento sobre o posto de coleta; em quais locais as embalagens de agroquímicos são descartadas e se é feita a tríplice lavagem da embalagens.

A escolha do agricultor foi aleatória, o único critério foi o produtor fazer parte da área de abrangência do município de Santarém. A principal dificuldade durante a realização das entrevistas foi o fato das comunidades e/ ou propriedades se encontrarem distantes um das outras.

Durante a visita foi possível notar irregularidades no manejo de defensivos agrícolas e foram transmitidas sugestões aos entrevistados sobre o descarte, proteção antes, durante e após a aplicação para não ocorrer futuras intoxicações de pessoas e animais, nem contaminação do meio ambiente, sendo está uma ação de caráter educativo.

\section{Análise de dados}

Os dados foram tabelados em planilhas do Microsoft Excel ${ }^{\circledR} 2017$, sendo posteriormente apresentados em forma de gráficos e tabelas para facilitar a discussão e entendimento do leitor.

\section{Resultados e discussão}

Neste item serão apresentados os resultados obtidos no estudo a partir da aplicação do questionário aos produtores de hortaliças. Os dados foram organizados seguindo a ordem das perguntas realizadas no questionário.
Durante o levantamento dos dados verificou-se que predominantemente $100 \%$ dos produtores respeitam o período de carência (período entre a última aplicação do agrotóxico e a reaplicação, colheita, uso ou consumo do alimento). Para Fernandes Neto e Sarcinelli (2009), os agroquímicos são contaminantes pela intensidade que são usados, pelo não cumprimento do intervalo e de dosagens fora das recomendações descritas pelo fabricante.

Quanto ao tipo de cultivo, 28 produtores $(93,3 \%)$ declararam realizar o preparo convencional e apenas 2 produtores $(6,7 \%)$ realizam o cultivo protegido. Isso pode ser explicado, devido ao cultivo convencional requer grandes quantidades de insumos, como fertilizantes e defensivos agrícolas para a sua manutenção e por muitas vezes esses agroquímicos são usados de maneira inadequada, gerando riscos ao meio ambiente (Rosset et al. 2014).

Na Figura 1 observa-se que $14(46,6 \%)$ produtores seguem as instruções para aplicação apresentadas no rótulo do produto, $8(26,6 \%)$ do vendedor, $4(13,4 \%)$ do engenheiro agrônomo ou técnico e $4(13,4 \%)$ seguem as orientações dos familiares. Seguindo as instruções do rótulo os produtores minimizam os riscos de toxicidade ao meio ambiente e níveis de poluentes, além da utilização adequada do produto. Castelo (2003) relata que devido à quantidade de informações técnicas e o tamanho das letras contidas na bula, muitos agricultores afirmam que leem as informações sobre dosagens e pragas, mas não dão a devida atenção sobre cuidados de segurança. Dados distintos foram evidenciados por Viero et al. (2016) em seu estudo realizado no interior do Rio Grande do Sul, que apontou que os produtores utilizavam os agroquímicos conforme receituário do engenheiro agrônomo das empresas fornecedoras dessas substâncias.

A maioria dos produtores $23(76,6 \%)$ afirmaram ter recebido orientações sobre o uso de agroquímicos, deste $9(29,9 \%)$ receberam instruções do técnico, $6(19,9 \%)$ do órgão de Assistência Técnica e Extensão Rural (ATER), $4(13,4 \%)$ do vendedor, $3(10 \%)$ de assistência particular e apenas $1(3,4 \%)$ recebeu instrução "familiar". No entanto 7 produtores, totalizando $23,4 \%$ relataram não receber orientações sobre a utilização de defensivos agrícolas. Corroborando com o estudo de Mendes et al. (2014), na qual a maioria dos entrevistados $(60,9 \%)$ receberam orientações de profissionais especializados.

Quando questionados sobre o tipo de agrotóxico utilizado em suas propriedades $12(40 \%)$ produtores relatam que fazem o uso apenas Inseticida, $6(20 \%)$ produtores utilizam Fungicida e Herbicida, 5 (16,7\%) aplicam Inseticida e Herbicida, $4(13,3 \%)$ utilizam Fungicida e Herbicida e apenas $3(10 \%)$ usam os três tipos de defensivos (Fungicida, Herbicida e Inseticida). Resultados semelhantes foram evidenciados por Mendes et al. (2014) no município de Tianguá, Ceará, acerca do uso e aplicação de agroquímicos, onde os inseticidas foram os mais utilizados pelos produtores. 
Figura 1 - Resposta dos produtores quando questionados sobre as instruções de aplicação.

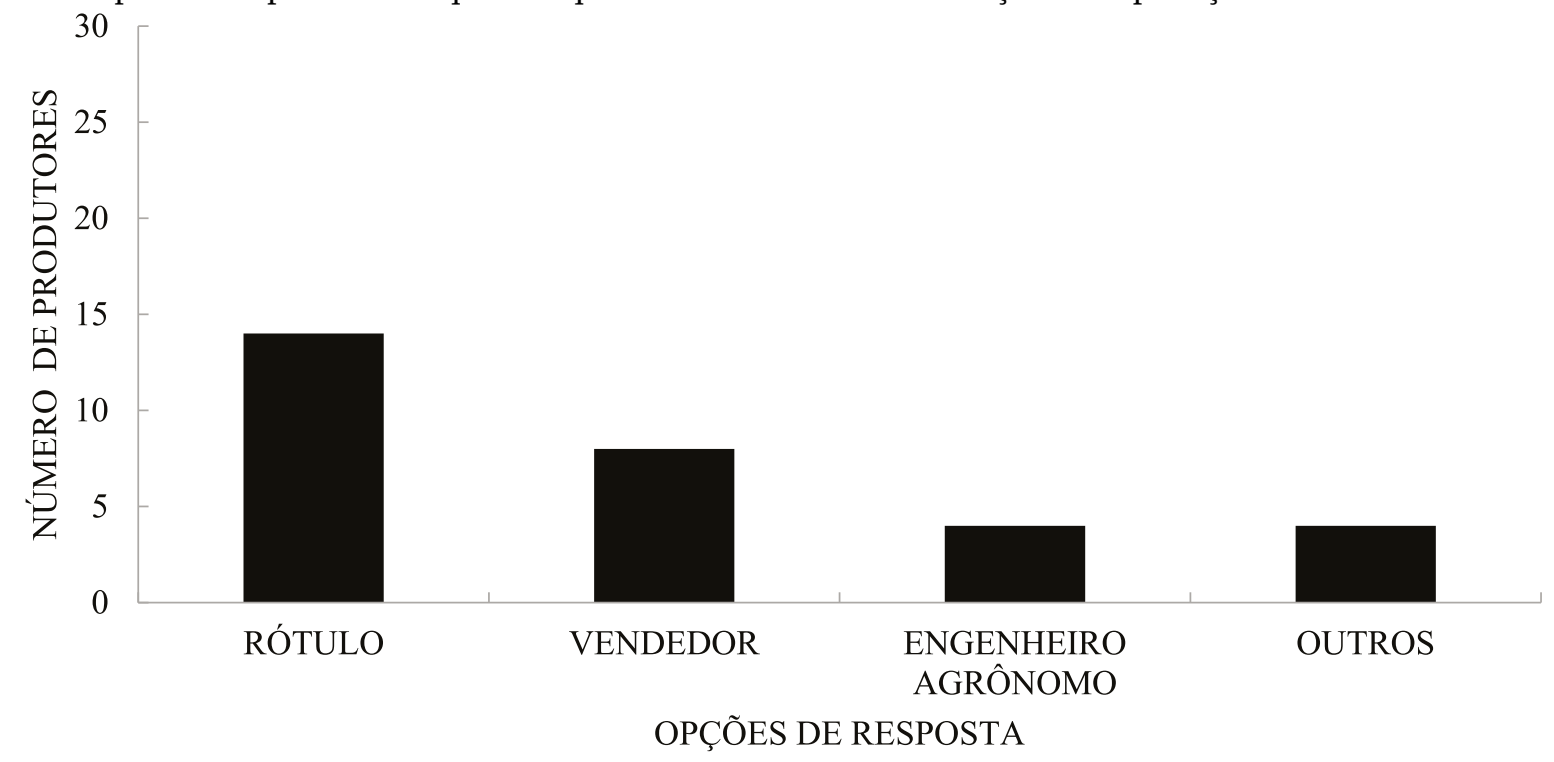

Na utilização de equipamentos de proteção individual, a maioria dos produtores 18 (60\%) fazem uso de utilizam EPI parcialmente, outra parte não utiliza e uma pequena parcela faz uso completo do EPI (Figura 2). Isso pode ser explicado, possivelmente, devido as condições financeiras que podem inviabilizar a comprar de EPIs, associado ao desconforto da utilização desses equipamentos em decorrência do calor intenso na região. Foi possível inferir que a maioria não utiliza EPI ou usa parcialmente, segundo Viero et al. (2016), os trabalhadores ficam expostos a contaminações e acidentes, cujo uso correto dos EPI poderia minimizar ou evitar. Santos et al. (2017) afirmam que entre todas as atividades de manejo das culturas, a aplicação de defensivos agrícolas é a que oferece maior perigo ao trabalhador rural, para Nunes (2010) a falta ou a utilização parcial do EPI representa grande perigo à saúde do aplicador, aumentando os riscos de intoxicações.

Figura 2 - Resposta dos produtores quanto a utilizam equipamento de proteção individual.

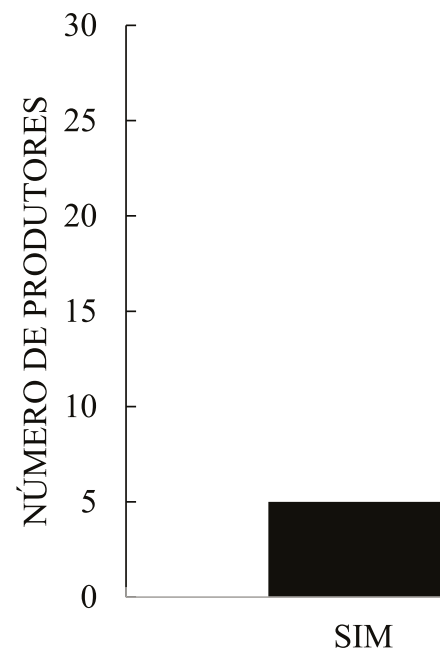

Dos produtores entrevistados que responderam fazer o uso parcial do equipamento de proteção individual, grande parte utiliza, principalmente, luvas (13), botas (13) e óculos (5), os demais equipamentos adotados estão apresentados na Tabela 1. Vale ressaltar que durante a entrevista uma das principais queixas pelo uso parcial do EPI é a falta de conforto térmico e o desconforto durante a utilização, resultados semelhantes foram encontrados no estudo de Santos et al. (2017) que realizaram a aplicação de um questionário na região do município de Governador Mangabeira-BA, os resultados obtidos mostraram que os principais motivos alegados pelos produtores para a não utilização ou utilização incompleta do EPI foi por estes serem considerados desconfortáveis, esquentarem demais (calor), dificultarem a respiração e mobilidade. 
Percepção de risco da utilização e descarte de embalagens de agroquímicos em hortas, em Santarém, Pará

Tabela 1 - Respostas dos produtores que utilizam EPI parcialmente.

\begin{tabular}{ccccccc}
\hline EQUIPAMENTOS & LUVAS & BOTAS & CHÁPEU & ÓCULOS & *MCM & *AI \\
\hline QUANTIDADE & 13 & 13 & 1 & 5 & 3 & 1
\end{tabular}

*Cada produtor pode selecionar mais de uma opção de acordo com a vestimenta Adotada.

*MCM = Macacão Com Mangas

*AI= Avental Impermeável

Predominantemente 29 (96,6\%) dos produtores afirmaram lavar a roupa após a aplicação e somente $1(3,4 \%)$ não realiza esse procedimento. Esse dado é importante, pois a lavagem das roupas reduz a fonte de contaminação. Em estudo semelhante realizado por Costa et al. (2017) na comunidade Rodeador no Cariri Cearense encontrou-se resultados divergentes, onde por pois a grande maioria dos entrevistados não lavam as roupas após o trabalho $(91,7 \%)$.

Em relação ao conhecimento sobre os postos de coleta, identificou-se que $22(73,4 \%)$ produtores não conhecem e não sabem sobre o posto de coleta e somente 8 $(26,6 \%)$ tem conhecimento sobre o local de devolução das embalagens vazias. Esse dado é preocupante, pois quando as embalagens vazias de agroquímicos são descartadas em locais inadequados, essas podem contaminar o meio ambiente, além de pode aumentar os riscos à saúde de seres humanos e animais. Nesse contexto, Cavalcante et al.
(2015) descrevem que muitos produtores desconhecem a à legislação (Lei no 9.974 de 2000) referente à devolução das embalagens vazias, que devem ser entregues no local de venda que o produtor efetuou a compra (artigo 60). Mendes et al. (2014) afirmam que as embalagens dos agroquímicos, são um problema de saúde pública, dessa forma, a destinação final correta realizada por meio da devolução no posto de coleta, busca reduzir o risco para a saúde das pessoas e de contaminação do meio.

A maioria dos produtores 18 (60\%) descartam, principalmente, as embalagens vazias de agroquímicos no lixo comum ou deixam próximo a cultura $4(13,4 \%)$, outros ambientes também são utilizados para o descarte conforme Figura 3. Teixeira et al. (2014) em estudo semelhante realizado na região de Guanambi-BA, identificaram que o descarte é realizado próximo a cultura, enterram, queimam e apenas um produtor devolve no posto de coleta.

Figura 3 - Resposta dos produtores em relação ao local de descarte das embalagens vazias de agrotóxico.

\section{DEVOLVE NO POSTO DE COLETA}

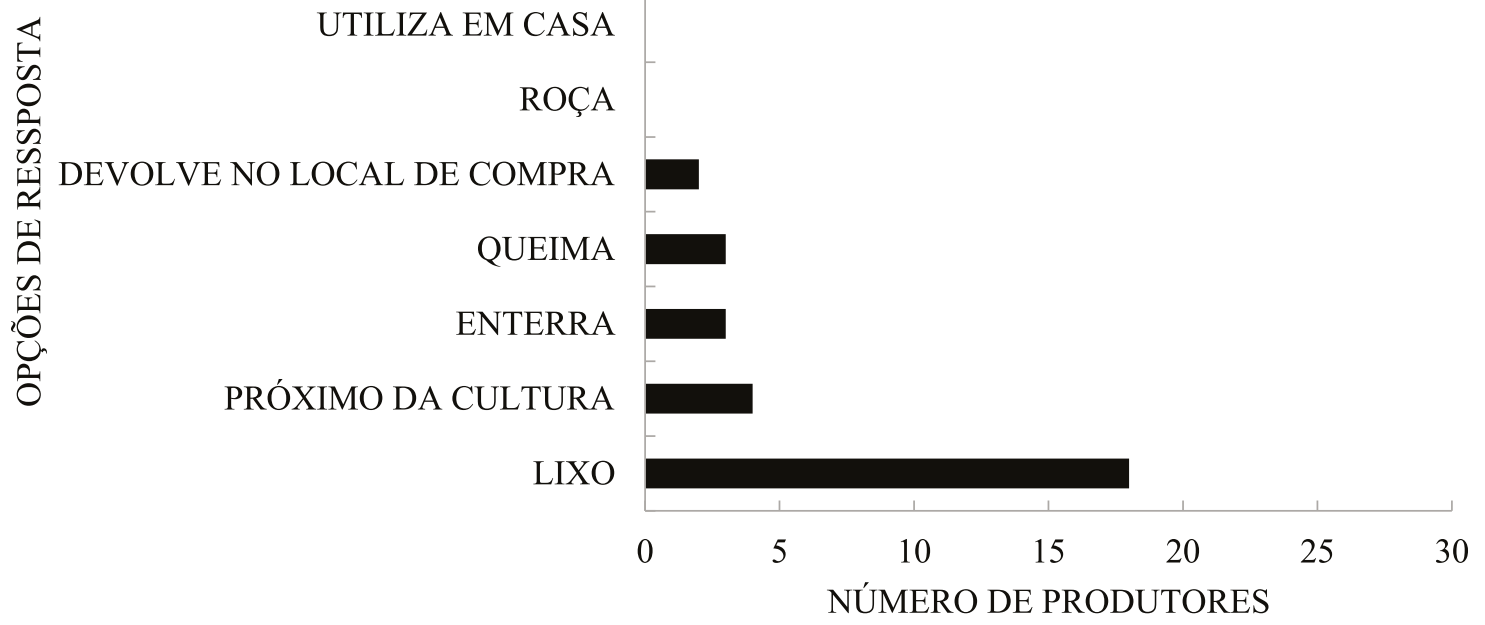

Questionados sobre a realização da Tríplice Lavagem, $24(80 \%)$ dos produtores não realizam esse procedimento em embalagens vazias, e a minoria de $6(20 \%)$ executam a lavagem das embalagens. A tríplice lavagem das embalagens vazias está previsto na legislação brasileira, (Brasil, 2002), e deve ser executado sempre antes do descarte da embalagem, evitando a contaminação do ambiente. Resultados semelhantes foram observados por Costa et al. (2017) em estudo no Cariri Cearense, no qual, $87,55 \%$ não realizam a tríplice lavagem das embalagens. Segundo Reinato et al. (2012) a embalagem vazia de defensivos agrícolas, retém quantidade do produto, e essa quantidade varia em função da superfície, do formato e da formulação, por isso a necessidade da execução da tríplice lavagem, assim diluindo resíduos do produto e consequentemente reduzindo possíveis contaminações e intoxicações.

\section{Conclusão}

Os produtores de hortaliças do município de Santarém, Pará. não fazem o descarte correto das embalagens vazias de agroquímicos, descartando-as em ambiente inadequado, sendo que nenhum produtor devolveu a 
Rebelo da Silva, E. B. et al.

embalagem no posto de coleto conforme preconiza a legislação brasileira. Além disso, os produtores seguem principalmente as instruções do rótulo, e recebem orientação de profissionais com conhecimento técnico sobre o assunto. Percebeu-se que o agroquímico mais adotado foi o inseticida. Por fim, apenas uma pequena parte dos produtores utiliza EPI completo e a maioria realiza a lavagem das roupas após a utilização, entretanto, não realizam a tríplice lavagem das embalagens vazias.

\section{Ética da pesquisa}

Este estudo seguiu os preceitos éticos de pesquisa, obedecendo às normas de sigilo confidencial de todas as propriedades visitadas "in loco", bem como os aspectos vinculados aos proprietários. Todos os participantes concordaram voluntariamente em participar da pesquisa, tendo sido informados sobre as questões de sigilo de dados. Ademais, registraram seu aceite mediante assinatura de termo de consentimento livre e esclarecido.

\section{Referências}

Alves, T. C. 2013. Manual de equipamento de proteção individual. Circular técnica. São Carlos, SP: Embrapa Pecuária Sudeste. Disponível em: < https://www.infoteca.cnptia.embrapa.br/bitstream/doc/975090/1/ Documentos111.pdf $>$.

Bernardi, A. C. A.; Hermes, R.; Boff, V. A. 2018. Manejo e Destino das embalagens de agrotóxicos. Perspectiva, Erechim, 42: 15-28. Disponível em: <http://www.uricer.edu.br/site/pdfs/perspectiva/159_719.pdf>.

Bolfarine, H.; Bussad, W. O. 2004. Elementos de Amostragem. Universidade de São Paulo, Instituto de Matemática e Estatística, São Paulo, Brasil.

Brasil. Decreto 4.074, de 04 de janeiro de 2002. Regulamenta a Lei 7.802 que dispõe sobre agrotóxicos e afins. [Brasil] Diário Oficial da União, 08 jan 2002; Seção I:1. Disponível em: < http://www.planalto. gov.br/ccivil_03/decreto/2002/d4074.htm $>$.

Castelo, B. M. 2003. Avaliação do conhecimento do rótulo dos inseticidas por agricultores em uma área agrícola do Distrito Federal. Horticultura Brasileira, 21: 570-73. Doi: https://doi.org/10.1590/ S0102-05362003000300031.

Cavalcante, M.; Santos, F.X.; Pereira, D. A.; Barbosa, T. J. A.; Silva Neto, J.V. 2015. Diagnóstico do uso de agrotóxicos por agricultores familiares de Maragogi/AL. Cadernos de Agroecologia, 10: 1-7. Disponível em: $<$ http://revistas.aba-agroecologia.org.br/index.php/cad/article/ view/17226>.

Costa, M. N. F; Souza, C. C.; Freitas, D. R.; Silva, L. M.; Santos, H, R. 2017. Levantamento da forma de uso dos agrotóxicos pelos agricultores na comunidade Rodeador no Cariri Cearense. Anais do Congresso Técnico Científico da Engenharia e da Agronomia. Belém, PA, Brasil. Disponível em: <https://www.confea.org.br/sites/default/files/antigos/ contecc2017/agronomia/84_ldfdudapancrncc.pdf $>$.

Fernandes Neto, M. L.; Sarcinelli, P. N. 2009. Agrotóxicos em água para consumo humano: uma abordagem de avaliação de risco e contribuição o processo de atualização da legislação brasileira. Engenharia Sanitária e Ambiental, 14: 69-78. Doi: https://doi.org/10.1590/S141341522009000100008 .

Ferreira, G. H. C.; Cepolini, E. I. S. 2014. Agroecologia, Alimentação e Saúde: um diálogo possível e necessário, Entremares, São Paulo, Brasil.

Lara, S. S.; Pignati, W. A.; Pignati, M. G.; Leão, L. H. C.; Machado, J. M. H. 2019. A agricultura do agronegócio e sua relação com a intoxicação aguda por agrotóxicos no brasil. Revista Brasileira de Geografia Médica e da Saúde, 15: 1-19. Doi: http://dx.doi.org/10.14393/Hygeia153246822.

Lopes, C. V. A.; Albuquerque, G. S. C. 2018. Agrotóxicos e seus impactos na saúde humana e ambiental: uma revisão sistemática. Saúde em Debate, 42: 518-534. Doi: https://doi.org/10.1590/01031104201811714.
Mendes, E. N.; Freire, J. E.; Figueiredo, M. F.; Braga, P. E. T. 2014. O uso de agrotóxicos por agricultores no Município de Tinguá-CE. Revista Agropecuária Científica no Semiárido, 10: 7-13. Doi: http://dx.doi. org/10.30969/acsa.v10i1.368.

Nunes, G. C. 2010. Uso do EPI - Equipamentos de Proteção Individual nas pequenas propriedades rurais produtoras de fumo no município de Jacinto Machado-SC. Criciúma (SC): Universidade do Extremo Sul Catarinense, 59 f. Especialização. Disponível em: < http://repositorio. unesc.net/bitstream/1/822/1/Gezziano\%20C\%C3\%B3rdova\%20Nunes. $\mathrm{pdf}>$.

Oliveira, M. M.; Sabonaro, D. Z. 2016. Logística reversa e o processo de destinação das embalagens vazias de agrotóxico. Revista da Universidade Vale do Rio Verde, 14: 377-383. Doi: http://dx.doi.org/10.5892/ruvrd. v14i2.2688.

Pantoja, T. F; Cesarino, F; Cruz, E. L; Vaz, J. D. C.; Sousa, T. G. 2013. A importância da horta escolar no processo ensino aprendizagem de botânica em uma escola de Macapá - AP. Anais do 64ํㅡ Congresso Nacional de Botânica, Belo Horizonte, MG, Brasil. Disponível em: <http:// plataforma.sacaca.ap.gov.br/pesquisador/1695/fabiano-cesarino > .

Reinato, R. A.; Garcia, R. B. G.; Zerbinatti, O. E. 2012. A situação atual das embalagens vazias de agrotóxicos no Brasil. Engenharia Ambiental, 9: 79-94. Disponível em: <http://ferramentas. unipinhal.edu.br/engenhariaambiental/include/getdoc. php id $=2374 \&$ article $=779 \&$ mode $=$ pdf $>$.

Rosset, J. S.; Coelho, G. F.; Greco, M.; Leonardo Strey, L.; Gonçalves Junior, A. C. 2014. Agricultura convencional versus sistemas agroecológicos: modelos, impactos, avaliação da qualidade e perspectivas. Scientia Agraria Paranaensis, 13: 80-94. Doi: https://doi.org/10.18188/sap. v13i2.7351.

Santos, A. O.; Borges-Paluch, L. R; Cerqueira, T. P. S.; Teles, A. L. B. 2017. Utilização de equipamentos de proteção individual e agrotóxicos por agricultores de município do recôncavo Baiano. Revista da Universidade Vale do Rio Verde, 15: 738-754. Doi: http://dx.doi.org/10.5892/ruvrd. v15i1.3309.

Sousa, J. A.; Feitosa, H. D. O.; Carvalho; C. M. De; Pereira, C. F.; Feitosa, S. D. O; Silva, S. L. 2016. Percepção dos produtores rurais quanto ao uso de agrotóxicos. Revista Brasileira de Agricultura Irrigada, 10: 976989. Doi: http://dx.doi.org/10.7127/rbai.v10n500484.

Teixeira, J. H. S; Guimarães, M. A. S; Cardoso, S. C. 2014. Uso e cuidados com agrotóxicos na região de Guanambi, BA. Enciclopédia Biosfera, 18: 437-445. Disponível em: <http://www.conhecer.org. br/enciclop/2014b/AGRARIAS/levantamento\%20do\%20uso.pdf $>$.

Viero, C. M.; Camponogara, S; Cezar-Vaz, M. R.; Costa, V. Z.; Beck, C. L. C. 2016. Sociedade de risco: o uso dos agrotóxicos e implicações na saúde do trabalhador rural. Escola Anna Nery, 20: 99-105. Doi: https:// doi.org/10.5935/1414-8145.20160014. 